Originalveröffentlichung in: Hausmann, Andrea (Hrsg.): Handbuch Kunstmarkt.

Akteure, Management und Vermittlung. Bielefeld: Transcript-Verlag 2014, S. 439-447

\title{
Der Kunstmarkt im Internet
}

\section{Hubertus Kohle}

Das Internet, in den Vorstufen eine militärische und in der Frühzeit eine wissenschaftliche Angelegenheit, hat in den letzten Jahren vor allem im kommerziellen Bereich für Furore gesorgt. Es hat den Musikmarkt umgekrempelt und die CD als Speichermedium an den Rand gedrängt, da Musiktitel jetzt in der wolkig sogenannten »cloud « gespeichert und daraus abgerufen werden. Es ist dabei, den Buchmarkt zu revolutionieren, wobei nur noch die Frage ist, wie groß das Stück sein wird, das sich der online-Handel, den man wenigstens zur Zeit mit dem Namen amazon identifiziert, aus dem Gesamtkuchen herausschneidet. Und es könnte auch für den Kunsthandel eine wichtige Rolle spielen, wobei die Ausgangsvoraussetzungen hier weniger günstig scheinen, auch wenn durch Wegfall der Mietkosten für die Galerieräume in häufig erstklassigen Innenstadtlagen schon einmal ein wichtiger Kostenfaktor hinfällig würde. Weniger günstig, weil der Kunsthandel hier dem Museum ähnelt: Wenn das Bibliothekswesen wohl auch deswegen die Digitalisierungsbemühungen entschieden ausgeprägter verfolgt als etwa das Museumswesen, dann deswegen, weil es im Buch sowieso mit einem Reproduktionsmedium zu tun hat, das Museum dagegen mit Originalen. So eben auch der Kunsthandel: Die in ihm angebotenen Objekte, welche für Idealisten ohnehin mit Geld nicht aufzuwiegen sind, gerade deswegen aber zuweilen hohe 8-stellige Verkaufspreise erzielen, dürften dem schnöden Massenmedium Internet tendenziell eher nicht anvertraut werden. ${ }^{1}$ Manches von dem, was in diesem Zusammenhang zu erfahren ist, mag die Vermutung bestätigen. Unverkennbar ist das müde Lächeln, das man bei dem typischen Kunsthändler hervorruft, der auf das Thema angesprochen wird. Das Interesse der Kunstgalerien, sich mit dem Internet einen neuen Verkaufskanal zu eröffnen, scheint begrenzt. ${ }^{2}$ Und

1 | Zumindest wird das Internet als Massenmedium wahrgenommen, obwohl es durch seine radikale Individualisierbarkeit genau genommen eigentlich genau das Gegenteil darstellt.

2 I amazon, das zuletzt in den Kunsthandel eingestiegen ist, berichtet, dass ein guter Teil der von der Firma angeschriebenen Galerien auf das Angebot, über die global äuBerst erfolgreich agierende Plattform zu verkaufen, noch nicht einmal reagiert hat (vgl. 
Versuche, trotzdem aktiv zu werden, sind in teilweise spektakulären Fehlinvestitionen gemündet. So etwa die Absicht eines der größten Auktionshäuser der Welt (Sotheby's) sich mit dem größten online-Auktionator Ebay zusammenzutun. Das Projekt kostete den altehrwürdigen englischen Betrieb 100 Millionen Dollar und wurde nach weniger als einem Jahr mit einer entwaffnenden Begründung des Auktionshaus-Chefs William Ruprecht eingestellt: »Es sind einfach weniger Menschen als erwartet bereit, im Internet exklusive Kunstwerke zu kaufen.« ${ }^{3}$

Aber auch, wenn die in anderen Bereichen erzielten prozentualen Verkaufsanteile im Internet, die etwa beim Buchhandel schon jetzt in einem immerhin niedrigen zweistelligen Bereich liegen, beim Kunsthandel wohl illusionär bleiben dürften, ist dessen jährlicher Gesamtumsatz von ca. 46 Milliarden Euro (in 2011) doch derartig gewaltig, dass auch bescheidene Anteile davon lukrativ sind. ${ }^{4}$ Selbst wenn davon langfristig nur ein Zehntel in den online-Markt abwandert (also weniger, als das, was schon jetzt im Buchmarkt erzielt wird), ist das immer noch eine ausgesprochen lohnenswerte Summe.

Geschätzt wird, dass der online organisierte Kunsthandel zur Zeit um jährlich $20 \%$ wächst. ${ }^{5}$ Nicht durch Zufall haben daher in den letzten 15 Jahren hunderte von Unternehmen in diesen neuen Markt investiert, ${ }^{6}$ so dass eventuell auch die Vermutung bestehen darf, dass Sotheby's einfach zu früh dran war. ${ }^{7}$ Der Geschäftserfolg dieser Neugründungen ist allerdings meistens nicht erkennbar, weil man Zahlen nur sehr zurückhaltend herausgibt. Wenn zuletzt auch amazon in den Kunstmarkt eingestiegen ist und nach dem Modell seines Partnerprogramms Galerieangebote über seine Internet-Suchmaschine vertreibt, so muss das aber aufhorchen lassen. ${ }^{8}$ Denn an der Fähigkeit des größten Internet-Händlers der Welt, den richtigen Zeitpunkt für ein solches Engagement zu finden, wird man nicht so leicht zweifeln dürfen. Und das Geschäftsmodell ist ja ebenso simpel wie genial: amazon fungiert nur als Vermittler und muss noch nicht einmal Lagerraum vorhalten. Und es ist als Marke derartig mächtig und universell geworden - universell insofern, als es praktisch alle Produktbereiche bedient -, dass auch

Aaron Souppouris, Amazon launches an online marketplace for fine art, www.theverge. com/2013/8/6/4593496/amazon-art-fine-art-marketplace, zuletzt besucht am 30.8 . 2013).

3 I Computerwoche vom 5.2.2003 (www.computerwoche.de/a/sotheby-und-039-s-stopptonline-versteigerungen-bei-ebay,536156, zuletzt besucht am 30.8.2013).

4 I Tefaf Art Market Report 2013, S. 21.

5 I Alex Hudson, Art ssold more online than in galleries،, in: www.bbc.co.uk/news/techno logy-23054641, zuletzt besucht am 30.8.2013.

6 | Vgl. ebd.

7 | Dabei sind einige der früh gegründeten Häuser wie artnet.com und artprice.com durchaus auch heute noch tätig.

8 | www.amazon.com/art, vgl. etwa www.skatepress.com/?cat=196 (zuletzt besucht am 30.8.2013). 
jemand, der oder die nach Kunst sucht, hier nachfragen dürfte. Das könnte im übrigen auch der entscheidende Vorteil gegenüber online-Kunsthändlern sein, die ein ähnliches Prinzip schon vorher verwirklicht haben (etwa artsy.net). Weniger gewichtig scheint dagegen die Tatsache, dass einige der schillerndsten Figuren des internationalen Milliardärs-Jetsets zuletzt Millionen in den online-Kunsthandel investiert haben. ${ }^{?}$

So wird es auch nicht verwundern, dass wenigstens für bestimmte Bereiche Untersuchungen vorliegen, die die Aussichten für den online-Kunsthandel durchaus positiv einschätzen. Eine Befragung des englischen Kunstversicherers hiscox, die sich an Händler für moderne und zeitgenössische Kunst richtete, hat ergeben, dass mehr als ein Drittel aller angesprochenen Galerien einen Anteil von $10-30 \%$ für möglich hält, und immerhin ca. ein Achtel glaubt, in ein paar Jahren mehr als die Hälfte des Umsatzes online zu machen. ${ }^{10}$ Für ältere Kunst sieht das sicherlich anders aus, hierfür aber liegen kaum seriöse Untersuchungen vor. Immerhin ist für bestimmte Bereiche der älteren Kunst (bzw. des Kunstgewerbes) das Geschäft inzwischen fast vollständig ins Internet abgewandert: Die im texanischen Dallas beheimatete Firma Heritage Auctions, die sich auf Münzen, Waffen und Juwelen spezialisiert hat, konnte zuletzt an die $85 \%$ ihres Geschäftes im Internet machen. ${ }^{11}$ Erstaunlich aber sind doch auch andere Zahlen: Mehr als zwei Drittel aller befragten Kunstkäufer hat z.B. schon einmal über das Internet Kunst gekauft (wie gesagt: moderne bzw. zeitgenössische Kunst). ${ }^{12}$ Dabei überrascht fast noch mehr, dass sie dies nur auf der Basis von digitalen Reproduktionen taten. Andererseits scheint eben letztere Tatsache dafür verantwortlich zu sein, dass in großen Teilen der Kundschaft doch auch Skepsis vorherrscht, da die Inaugenscheinnahme des Originals für wesentlich gehalten wird. Es steht zu vermuten, dass der Anteil der Skeptiker mit dem Alter der Kunst wächst, allerdings nicht mit dem Alter der Käufer. So wurde von Hiscox herausgefunden, dass ein guter Teil der online-Käufer schon zur Generation $65+$ gehört. ${ }^{13}$

Nicht überraschend ist die Tatsache, dass der untere Preisbereich im onlineKunstgeschäft dominiert: Fast $80 \%$ aller Transaktionen betreffen Objekte im

9 | Vgl. Georgina Adam, Buying art online: A click away from a masterpiece?, www.bbc. com/culture/story/20130625-a-clickaway-from-a-masterpiece (zuletzt besucht am 30.8. 2013).

101 Hiscox. The Online Art Trade, 2013, S. 4 (www.hiscox.com/ /media/Files/H/Hiscox/ content-pdf/Online-Art-Trade-Report-UPDATED-FINAL.pdf, zuletzt besucht am 30.8.2013). 11 | www.ha.com/, vgl. Georgina Adam (wie Anm. 9). Neben kunsthandwerklichen Objekten bietet die Firma aber durchaus auch Dinge wie teure Weine oder Hermes-Taschen an. Der Übergang ins Luxus-Geschäft ist hier also fließend. Offenbar haben Kunstwerke und Luxusobjekte die gleiche Funktion, nämlich diejenige, Distinktionsmerkmale der Oberklasse zu sein.

12 | Hiscox (wie Anm. 9), S. 3.

13 | Ebd. 
Wert von unter $10.000 £$. Firmen wie paddle8 spezialisieren sich dann auch gleich auf den Preisbereich unter $100.000 \$ .{ }^{14}$ Das ändert nichts daran, dass der Wettlauf um Höchstpreise auch das online-Kunstgeschäft erfasst hat. Die österreichisch geführte, in Berlin angesiedelte »Galerie« Auctionata hat zuletzt einen Egon Schiele für fast 2 Millionen Euro versteigert. Ob der Käufer auch hier das Bild vorher nicht im Original gesehen hat, ist nicht bekannt. Stolz ist das Haus jedenfalls nicht nur auf den Rekord, sondern darauf, dass der gesamte Ablauf einer Versteigerung ins Internet transloziert wurde..$^{15}$

In der longue durée betrachtet, verschärft der Internet-Kunsthandel eigentlich nur eine Tendenz, die auch schon in der offline-Welt eingesetzt hat. Zu nennen ist in diesem Zusammenhang der Name Alfred Taubmans, eines amerikanischen shoppingmall tycoons, der in den frühen 1980 or Jahren Sotheby's kaufte und ganz im Stil der Warenhaus-Apologeten versuchte, die Zugangsbarrieren eines entschieden snobistischen Luxus-Etablissements wie dem des traditionellen Kunsthandels abzubauen. $\mathrm{Zu}$ seinen vielfältigen Strategien gehörte auch die Produktion von farbenprächtigen, wortreich ausgestatteten Verkaufskatalogen, die geeignet waren, eine weniger exklusive und auch weniger kennerschaftliche, dabei nichtsdestoweniger wohlhabende Käuferschicht anzusprechen. Man wird vermuten dürfen, dass hier auch Personen interessiert werden sollten, die im Segment des neuen Geldes angesiedelt waren, während der traditionelle Kunsthandel eher vom alten Geld dominiert war. $\mathrm{Zu}$ diesen neuen Käuferschichten gehören selbstverständlich auch die Globalisierungsgewinner in den Schwellenländern, von Russland über China bis in Teile Südamerikas, deren Kunstmärkte insgesamt zu den sich am dynamischsten entwickelnden zählen. Generell aber kommt an der Stelle auch eine Tendenz zum Ausdruck, breitere Käuferschichten einzubeziehen, ${ }^{16}$ also solche, die weder über neues noch über altes, sondern einfach nur über wenig Geld verfügen. Diese Entwicklung »Demokratisierung « zu nennen, ist zwar nicht so ganz falsch, die sublimen Konzepte, die mit dem Begriff assoziiert werden, sollte man aber eher zurückhaltend verwenden. Vielleicht wäre der weniger emphatische Begriff der »Nivellierung « adäquater.

Kunstinstitutionen - seien sie als Museum dem öffentlichen oder als Galerien dem privaten Bereich zuzuordnen - sind allen Veränderungen der letzten Jahrzehnte zum Trotz weiterhin von einer gewissen Exklusivität geprägt, die die vir-

14 | Vgl. Adam, wie Anm. 8.

15 | Vgl. www.presseportal.de/pm/101395/2499812/auctionata-bricht-online-rekordegon-schieles-liegende-frau-fuer-eur-1-8-millionen-live-versteigert (zuletzt besucht am 30.8.2013). Für einen Edward Hopper hat es bei einer Auktion bei Christies' auch schon einmal 10 Millionen Dollar gegeben. Das Gebot wurde über das Internet abgegeben.

16 | In die gleiche Richtung dürfte amazon mit der Absicht zielen, das Kunstgeschäft zu entmystifizieren. Vgl. tdz, Kunst für alle, in: www.internetworld.de/Nachrichten/E-Com merce/Handel/Marktplatz-Amazon-Art-eroeffnet-Kunst-fuer-alle-78667.html (zuletzt besucht am 30.8.2013). 
tuellen Zugangsbarrieren einigermaßen hoch erscheinen lässt. Es spricht vieles dafür, dass diese Institutionen auf Dauer besser überlebensfähig sind, wenn sie sich darum bemühen, diese Barrieren abzusenken - und dies insbesondere auch über den Einsatz von social media angehen. Eine Galerie zu betreten, erfordert für den habitué nicht viel, der Durchschnittsmensch muss sich aber überwinden. Das fällt im Internet weg. Abschätzige Blicke und herablassende Ansprachen sind hier nicht zu erwarten, zudem ist die Auswahl natürlich viel größer als in einer echten Galerie. Das hat im übrigen auch eine Konsequenz, die auf eine systematische Ermächtigung des Kunden gegenüber dem Verkäufer hinausläuft. Denn die Preisvergleichsmöglichkeiten verbessern sich jetzt exponentiell. Und auch, wenn im Unterschied etwa zum antiquarischen Buchmarkt, wo nur im Erhaltungszustand unterschiedliche Exemplare eines Buches nebeneinanderstehen und sich allzu deutliche Preisunterschiede verbieten, ein Kunstwerk immer ein unnachahmbares Einzelwerk ist: Die Möglichkeiten des Käufers, einen Galeristen mit dem Hinweis auf ganz ähnliche Werke des gleichen Künstlers in deutlich niedrigerem Preisbereich unter Druck zu setzen, häufen sich. ${ }^{17}$ Dabei haben die technischen Möglichkeiten zuweilen einigermaßen abstruse Konsequenzen: So können bei artsy (www.artsy.net) die Werke nach allen möglichen Kriterien gefiltert werden, unter anderem auch nach der Farbe, die bequem aus einem Farbspektrum heraus anzusteuern ist. Es werden dann nur solche Arbeiten ausgegeben, die einen größeren Anteil dieser Farbe aufweisen können. Das, was beim Kunstkenner Belustigung verursacht, dürfte andererseits aber nur ein meist verschwiegenes Kaufkriterium bezeichnen, denn wie oft hört man von Menschen, die sich die Kunstwerke an der Wand passend zur Farbe der Tapete und der Möbel aussuchen! Man wird deswegen mit einem gewissen Recht (und hoffentlich ohne der Herablassung geziehen zu werden) von einer Dilettantisierung des Kunstgeschäfts reden dürfen.

Der online-Markt gestaltet inzwischen sämtliche Bereiche des Kunsthandels mit. Neben den online nachgebildeten Auktionen, die wiederum mit den im Hochpreis-Bereich wichtigen Telefonbietern immer schon virtuelle Vorläufer hatten, gibt es das web-gestützt simulierte Galeriegeschäft. Daneben denjenigen Unternehmer, der wie amazon ein Vermittlergeschäft zu den Galerien betreibt und zudem die Direktvermittler. Außerdem sind alle möglichen Zwischenformen und Doppelstrategien zu beobachten. Christie's kennt die Kombination von klassischer und online-Auktion, aber auch online-only, von denen die altehrwürdige Firma in 2013 wöchentlich durchschnittlich eine ausrichten will. ${ }^{18}$ Die Direktvermittler dürften dabei zumindest für die Künstler die auf den ersten Blick interes-

17 | Vgl. zu diesem wichtigen Punkt den Kommentar von Isa Bickmann auf http://blog. arthistoricum.net/beitrag/2013/08/19/kunsthandel-online/\#commentList (zuletzt besucht am 30.8.2013).

18 | Vgl. Kunsthandel: Kunst online kaufen wird immer beliebter. Online Art Trade Report, www.artinfo24.com/kunstmarkt/news-1192.html (zuletzt besucht am 30.8.2013). 
santeste Variante darstellen: Nach dem Muster des Modells von James Jarvis', der in seinem berühmt gewordenen Buch »What would google do? « einen mit dem Internet auf allen Ebenen einsetzenden Niedergang des traditionellen Vermittlers postuliert hatte (vom Buchhändler über den Wohnungsmakler bis hin zum Lebensmittelverkäufer) funktioniert hier Kunsthandel unter Ausschaltung des Galeristen. ${ }^{19}$ Das heißt nicht, dass der ganze Verkaufserlös beim Künstler landet. Im bekanntesten Fall, saatchionline (www.saatchionline.com), bekommt der Betreiber der Website, der ja in gewisser Weise auch noch ein Vermittler ist, $30 \%$ des Erlöses. Anfänglich hatte Charles Saatchi, dessen Hass auf den gängigen Kunstbetrieb legendär ist, sogar auf jeden Gewinn seinerseits verzichtet. Und hierhin gehören auch Unternehmungen, die ganz neue Vermittlungsmodelle geschaffen haben, welche ohne das Medium Internet eigentlich nur schlecht vorstellbar sind. ArtViatic (www.artviatic.com) ist ein erst 2012 gegründeter Anbieter, der ganz auf Exklusivität setzt und als eine Art peer to peer network arbeitet. Dabei werden Anbieter und Käufer, die einen hohen jährlichen Betrag zahlen müssen, um in dieses Netzwerk eingebunden zu werden, zwecks Vermittlung von Kunstwerken, die von 150.000 Euro an aufwärts kosten, direkt miteinander in Verbindung gesetzt. Das Modell ist auch deswegen interessant, weil es innerhalb des als Massenmedium verschrienen Internets einen Raum des Besonderen restituiert, der in gewisser Weise die Aura des klassischen Kunsthandels konserviert - wenn auch unter ganz anderen Bedingungen.

Denn ob andererseits die Jarvissche These im Falle von Kunstwerken so richtig greift, kann durchaus bestritten werden. Kunst ist eben doch mehr als Ware, mehr wenigstens als, sagen wir einmal, eine schnöde Digitalkamera. Sie bezieht ihr Faszinosum aus der Tatsache des Einmaligen und Exklusiven und bedarf entsprechender Pflege. Dazu gehört das intensive Gespräch mit einem Kenner oder einer Kennerin, als die die Galeristen fungieren könnten. Vielleicht auch die Inszenierung in einem gediegenen Ambiente, wobei gegen letzteres wiederum die Tatsache spricht, dass Galerien auch in der »realen Welt« einen großen Teil ihres Umsatzes auf Kunstmessen machen. ${ }^{20}$ Daneben und besonders wichtig: Die aktive Bewerbung und Pflege des Künstlernamens, ohne die ein Werk mit einer gewissen Wahrscheinlichkeit im Wust der hunderttausendfachen Angebote unentdeckt bleibt. Und vor allem natürlich: Der Echtheitsnachweis, der von Häusern wie dem erwähnten auctionata durch Hinzuziehung eines mehrhundertköpfigen Expertengremiums garantiert werden soll. ${ }^{21}$ Erst wenn diese - und sicherlich noch manche anderen - Faktoren stimmen, besitzt ein Kunsthänd-

19 | Vgl. Jeff Jarvis, Was würde Google tun?: wie man von den Erfolgsstrategien des Internet-Giganten profitiert, München 2009 (zuerst englisch 2009).

20 | Vgl. Hudson, wie Anm. 4.

21 | Vgl. www.focus.de/digital/internet/dld-2013/digital-star/digital-star-perspectiveauctionataden-kunsthandel-online-bringen_aid_899629.html (zuletzt besucht am 30.8. 2013). 
ler das Renomee, das er braucht, um am Markt bestehen zu können. Vertrauen dürfte ein Wert sein, der im Kunsthandel eine größere Rolle spielt als in allen anderen Bereichen. Dieses Vertrauen aufzubauen aber benötigt Jahre und Jahrzehnte. Manche der erfolgreichsten Galerien und Auktionshäuser sind ja sogar Jahrhunderte alt!

Andererseits bietet das Netz und vor allem das interaktive Web 2.0 eine Reihe von Funktionen, die manche der genannten Prozesse simulieren oder sogar besser, auf breiterer Publikumsbasis organisiert durchführen können. Dazu gehören natürlich auch die aus vielen anderen Bereichen bekannten Bewertungsmöglichkeiten, mit denen ein Kunde seine Zufriedenheit mit einem Unternehmen registrieren kann. Die Strategien, mit denen der online-Kunsthandel darüber hinaus einerseits effektiver zu funktionieren, andererseits die Aura des alten Kunstbetriebs zu übernehmen trachtet, sind in den WebPräsenzen der unterschiedlichen Anbieter zu erkennen.

Saatchionline steht an dieser Stelle für das Genre des Direktvermittlers. Künstler können hier ohne weitere Hürde ihre Werke hochladen und einem weltweiten Publikum zum Kauf anbieten. Rebecca Wilson, die bei Saatchi selbst eine einflussreiche Rolle innehat, aber auch die online-Variante mitbetreibt, hat ohne falsche Bescheidenheit dazu gesagt, man verkaufe dort »more in a month online than most bricks-and-mortar galleries sell in a year. ${ }^{22}$ Interessant ist vor allem die Abteilung »Collections«. Der riesigen Masse der Einzelwerke (es sollen um die 200.000 sein $^{23}$ ) soll mit diesen Collections eine gewisse Struktur vermittelt werden, die das Angebot vom Vorwurf des Massenlagers befreit. Die »Collections sind Zusammenstellungen von Kunstwerken nach bestimmten formalen oder inhaltlichen Kriterien, die in erster Linie von kunsthistorisch geschulten Mitarbeitern von saatchionline vorgenommen werden. Damit führt der Anbieter eine Bewertungskomponente ein, die dem Angebot die Beliebigkeit nimmt und ihm gleichsam Sinn zuweist. Die Gestalt solcher »Collections « dürfte allerdings Traditionalisten eher abschrecken. Denn wenn hier »Collections inspired by Kandinsky« eingerichtet werden, dann scheint doch einer billigen Oberflächenphänomenologie gehuldigt, die zwar viel über gängiges Kunstverständnis aussagt, aber jede tiefere künstlerische Identität missachtet.

Paddle8 (http://paddle8.com/) versteht sich als Auktionshaus, das Kunstwerke von »international renommierten Künstlern« versteigert. Wie die meisten anderen online-Kunsthänder auch, bedient es sich extensiv der sozialen Medien. Auf twitter hat es inzwischen über 8000 follower, das heißt einen Kanal, auf dem es Interessierte zwanglos kontaktieren und Geschichten zu den von der Galerie vertretenen Künstlern anbieten kann. Auch dies kann man zwanglos als einen Versuch der Sinnvermittlung verstehen. Andere Anbieter schaffen hier noch erheblich mehr, etwa artsy, das mehr als 50.000 follower zu bieten hat. Zudem gibt 
es ein weblog, auf dem ebenfalls locker über gehandelte Künstler berichtet wird. Mit einer Funktion wie »most viewed « kann sich der Interessierte sicher sein, das zu kaufen, was mainstream ist, »ending soon « erinnert an profane Verkaufsplattformen wie ebay, das übrigens ebenfalls Kunst im Angebot hat. Denn bei ebay wird alles versteigert, wieso nicht auch Kunst?

So zwiespältig das Bild in der Summe auch ist: Es besteht keinerlei Anlass, den online organisierten Kunsthandel zu verharmlosen oder sich gar über ihn lustig zu machen. Davor sollte schon der Erfolg von amazon warnen, das am Anfang noch belächelt wurde, inzwischen aber als so bedrohlich empfunden wird, dass das Ende einer kultivierten Welt unmittelbar bevorzustehen scheint. Wichtiger als der Gegensatz von online- und offline Kunsthandel dürfte in jedem Fall die Vielfältigkeit der Anbieter sein - ob im Internet oder in the real world. Dass diese im Internet eher bedroht ist, zeigt eben gerade amazon, das sich immer mehr zum Monopolisten entwickelt. Es liegt auch am Kunsthandel selber, dieser Entwicklung durch möglichst vielfältige Geschäftsmodelle entgegenzuwirken. Erst dann nämlich wird eines der großen Versprechen des Internets Wirklichkeit: jedem Interessierten eine Möglichkeit zu bieten, dem individuellen Geschmack gemäß Kunst zu rezipieren.

\section{LITERATUR}

Adam, Georgina: Buying art online: A click away from a masterpiece? (www.bbc. com/culture/story/20130625-a-clickaway-from-a-masterpiece).

Anonym, Kunsthandel: Kunst online kaufen wird immer beliebter. Online Art Trade Report, (www.artinfo24.com/kunstmarkt/news-1192.html).

Anonym, Sotheby's stoppt Online-Versteigerungen bei Ebay (www.computerwoche.de/a/sotheby-und-039-s-stoppt-online-versteigerungen-bei-ebay,536156).

Anonym, Skate's market notes (www.skatepress.com/?cat=196).

Anonym, Auctionata bricht Online-Rekord (www.presseportal.de/pm/101395/ 2499812 /auctionata-bricht-online-rekord-egon-schieles-liegende-frau-fuer-eur1-8-millionen-live-versteigert).

Anonym, Digital Star Perspective: Auctionata (www.focus.de/digital/internet/dld2013/digital-star/digital-star-perspective-auctionataden-kunsthandel-onlinebringen_aid_899629.html).

Bickmann, Isa: Kommentar auf http://blog.arthistoricum.net/beitrag/2013/08/19/ kunsthandel-online/\#commentList.

Hiscox. The Online Art Trade, 2013 (www.hiscox.com/ /media/Files/H/Hiscox/ content-pdf/Online-Art-Trade-Report-UPDATED-FINAL.pdf).

Hudson, Alex: Art >sold more online than in galleries` (www.bbc.co.uk/news/tech nology-23054641).

Jarvis, Jeff: Was würde Google tun? : wie man von den Erfolgsstrategien des Internet-Giganten profitiert, München 2009. 
Souppouris, Aaron: Amazon launches an online marketplace for fine art (www. theverge.com/2013/8/6/4593496/amazon-art-fine-art-marketplace). tdz, Kunst für alle (www.internetworld.de/Nachrichten/E-Commerce/Handel/ Marktplatz-Amazon-Art-eroeffnet-Kunst-fuer-alle-78667.html). Tefaf Art Market Report 2013. 